\title{
Challenges of atypical matrix effects in tissue
}

"The adverse impacts of matrix effects in tissue analysis may be far reaching and certain factors that have no effect on a plasma method may be very important to a tissue method."

Keywords: matrix effects $₫$ signal enhancement $\approx$ signal suppression $₫$ tissue analysis $₫$ tissue matrix

Matrix effects, which suppress or enhance ionization efficacy due to the presence of co-eluting substances, were first reported in 1993 by Kebarle and Tang [1] and were recently reviewed in 2009 by Côté et al. [2]. Matrix effects are observed during bioanalysis using LC-MS/MS. At first glance, there are not many differences between matrix effects in plasma and tissue. The methodologies for assessing matrix effects apply to both. The adverse impact to the method precision and accuracy have been reported for both [3]. Furthermore, the strategies for reducing and eliminating matrix effects are similar for both [4]. Because of all those similarities, we usually treat matrix effects in tissue similar to plasma underestimating the challenges in the process. Subsequently, matrix effects related problems in tissue analysis are usually discovered much later, require unplanned resources and cause unexpected setbacks in timelines.

In truth, there are significant differences between matrix effects in plasma and tissue. Assessing matrix effects in tissue poses more challenges, such as the presence of interference and endogenous compounds in blank matrix, the lack of correlations between the results of matrix effects in QC samples and those in study samples, and the lack of sufficient amount of blank matrix. The impacts of matrix effects in tissue samples are in general much more significant and difficult to manage. Tissue methods are often a little more variable and 'touchy' than plasma methods. Reducing and eliminating matrix effects in tissue often proves to be a much more challenging and laborious process. If we consider the assessment and reduction of matrix effects in plasma as 'typical', then assessment and reduction of matrix effects in tissue are 'atypical'. They warrant much more attention during method development and sample analysis.

One of the reasons that we underestimate the challenges of matrix effects in tissue is that we fail to fully appreciate the diversity and complexity of tissue matrices. In the rat, for example, there are three common types of systematic fluids available, whole blood, plasma and serum. All of these are homogenous and only one of them is collected and analyzed for a particular study. Think of the tissue matrices from the same rat; they may be whole tissues, such as the brain, heart, liver, kidney, muscle and so on, or parts of a tissue, such as the iris, cornea, retina, and sclera of the ocular tissue, cerebral cortex, cerebellum, hippocampus, hypothalamus or pre-optic area of the brain. There could be 10, 20 or even more types of tissues collected and analyzed, and each one of them will be far more complex than plasma since it is a complex and fully functional system. They are rich in cells, membranes, electrolytes, enzymes and transporters. They are heterogeneous (unlike plasma) and contain subsections with different chemical, physical and physiological properties. Because of tissue diversity and complexity, it is not uncommon to observe signal suppression for a compound in one tissue, but signal enhancement for the same compound in another tissue using the same extraction procedure and LCMS/MS conditions [5-7]. In fact, matrix effects are analyte- and tissue-dependent and there is no known pattern to predict the outcome. This point is illustrated by the work of Lv, who evaluated the matrix effects of morinidazole and its IS, metronidazole, in 24 matrices, of which 21 were tissue matrices [8].

When assessing and evaluating the matrix effects in tissue $[2,9,10]$, there is often a need to vary the methodologies to a certain extent. Carefully planning and executing the experiments to assess matrix effects may be important to obtain the proper conclusion and critical to the success of tissue analysis. For example, it may be necessary to subtract the response of interference or endogenous level in blank matrix when using the post extraction spike method [11]. Because of greater lot-to-lot variation for

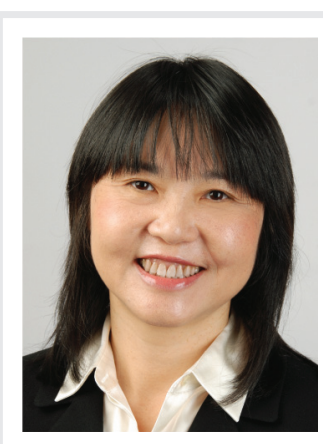

Stacy Ho

DSAR, Sanofi US, 153 Second Ave, Waltham, MA 0245I, USA

E-mail: stacy.ho@genzyme.com 
tissues, it is particularly helpful to evaluate the results of matrix effects in tissue in conjunction with those of selectivity [12], if multiple lots of blank matrix are available. While assessing matrix effects for a plasma method is typically a one-time event, it is a good idea to monitor the method performance during the entire course of sample analysis and expect 'surprises' caused by matrix effects for tissue methods. This is because an inherent challenge for tissue method development and qualification, is the binding and distribution of analyte in standard and QC samples, which does not closely mimic those of actual study samples. Such disparity means the lack of matrix effects for QC samples does not always translate to that of the study samples. For example, 7-aminoclonazepam was used as an IS for the determination of serotonin-selective antidepressants in liver and matrix effects were within $90-110 \%$ during method validation, which indicated minimal matrix effects. However, it had to be later replaced by a different IS due to unexpected matrix effects observed during sample analysis [13].

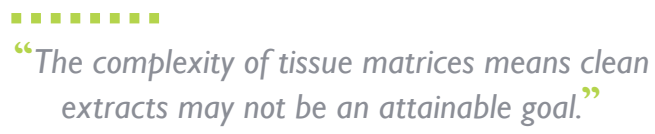

"The complexity of tissue matrices means clean extracts may not be an attainable goal."

$\square \square \square \square \square \square \square \square$

The adverse impacts of matrix effects in tissue analysis may be far reaching and certain factors that have no effect on a plasma method may be very important to a tissue method. Zhang et al. reported the change of reconstitution solvent from methanol to $60: 40$ (v:v) acetonitrile:methanol reduced matrix effects by approximately $10 \%$ for rat brain homogenate, but yielded no change for rat plasma [14]. Heller reported that method accuracy could not be replicated because ion suppression of the IS could not be reproduced when a bovine liver method was transferred to different laboratories, even when the same instrument was used [15]. Some tissue matrix components have a high tendency to retain on the column and need to be purposely washed out at the end of separation to avoid ion suppression of subsequent injections
$[16,17]$. While matrix effects are normally associated with LC-MS/MS methods, matrix effects in tissue analysis using GC-MS/MS have also been reported [18].

To reduce and eliminate matrix effects in tissue, commonly employed strategies such as improving sample clean-up, using stable-isotope labeled IS, or optimizing chromatographic and mass spectrometric conditions, may not be as effective as they are for plasma [4]. The complexity of tissue matrices means clean extracts may not be an attainable goal. Dodder reported the analysis of flame retardants, hexabromocyclododecane enantiomers, in marine tissues. The sample preparation consists of pressurized fluid extraction, removal of lipid and other interferences by gel permeation chromatography, followed by removal of potential ionization interferences by solid phase extraction [19]. Based on our experience with the plasma matrix, this kind of sample preparation procedure would yield very clean extract. However, in this case, the matrix effects were still observed under the chromatography conditions with stable-isotope-labeled IS. For plasma methods, one of the aforementioned approaches for the reduction of the matrix effects is sufficient in most cases. But for tissue methods, the most effective strategy is to take a comprehensive approach, such as optimizing all aspects of the method from sample extraction to detection.

In conclusion, there are great differences between matrix effects in tissue and in plasma. Compared with plasma, matrix effects in tissue are atypical. They need to be evaluated and addressed properly, with care and attention for successful tissue analysis.

\footnotetext{
Financial \& competing interests disclosure The author has no relevant affiliations or financial involvement with any organization or entity with a financial interest in or financial conflict with the subject matter or materials discussed in the manuscript. This includes employment, consultancies, honoraria, stock ownership or options, expert testimony, grants or patents received or pending, or royalties.

No writing assistance was utilized in the production of this manuscript.
}

\section{References}

1 Kebarle P, Tang L. From ions in solution to ions in the gas phase - the mechanism of electrospray mass spectrometry. Anal. Chem. 65(22), 972A-986A (1993).

2 Côté C, Bergeron A, Mess J-N, Furtado M, Garofolo F. Matrix effect elimination during
LC-MS/MS bioanalytical method development. Bioanalysis 1(7), 1243-1257 (2009).

3 Matuszewski BK, Constanzer ML, Chavez-Eng CM. Matrix effect in quantitative LC-MS/MS analyses of biological fluids: a method for determination of finasteride in human plasma at picogram per milliliter concentrations. Anal. Chem. 70(5), 882-889 (1998).

4

Chambers E, Wagrowski-Diehl DM, Lu Z, Mazzeo JR. Systematic and comprehensive strategy for reducing matrix effects in 
LC-MS/MS analyses. J. Chromatogr. B Analyt. Technol. Biomed. Life Sci. 852(1-2), 22-34 (2007).

5 Wu Y-T, Huang C-M, Lin C-C et al. Determination of melamine in rat plasma, liver, kidney, spleen, bladder and brain by liquid chromatography-tandem mass spectrometry. J. Chromatogr. A 1216, 7595-7601 (2009).

6 Ding Y, Lu CT, Yang J et al. Application of a liquid chromatography-tandem mass spectrometry (LC-MS/MS) method to the pharmacokinetics, tissue distribution and excretion studies of felotaxel (SHR110008) in tumor-bearing mice. J. Chromatogr. B Analyt. Technol. Biomed. Life Sci. 887-888, 61-66 (2012).

7 Tolgyesi A, Sharma VK, Fekete S, Lukonics D, Fekete J. Simultaneous determination of eight corticosteroids in bovine tissues using liquid chromatography-tandem mass spectrometry. J. Chromatogr. B Analyt. Technol. Biomed. Life Sci. 906, 75-84 (2012).

8 Lv W. Investigation of the effects of 24 bio-matrixes on the LC-MS/MS analysis of morinidazole. Talanta 80(3), 1406-1412 (2010).

9 Matuszewski BK, Constanzer ML, ChavezEng CM. Strategies for the assessment of matrix effect in quantitative bioanalytical methods based on HP-LC-MS/MS. Anal. Chem. 75(13), 3019-3030 (2003).
10 Matuszewski BK. Standard line slopes as a measure of a relative matrix effect in quantitative HPLC-MS bioanalysis. J. Chromatogr. B Analyt. Technol. Biomed. Life Sci. 830, 293-300 (2006).

11 Krijt J, Duta A, Kozich V. Determination of S-Adenosylmethionine and S-Adenosylhomocysteine by LC-MS/MS and evaluation of their stability in mice tissues. J. Chromatogr B Analyt. Technol. Biomed. Life Sci. 877(22), 2061-2066 (2009).

12 Lehtonen M, Storvik M, Malinen $\mathrm{H}$ et al. Determination of endocannabinoids in nematodes and human brain tissue by liquid chromatography electrospray ionization tandem mass spectrometry. J. Chromatogr. B Analyt. Technol. Biomed. Life Sci. 879, 677-694 (2011).

13 Butzbach DM, Stockham PC, Kobus HJ et al. Stability of serotonin-selective antidepressants in sterile and decomposing liver tissue. J. Forensic Sci. 58(Suppl. 1), S117-S125 (2013).

14 Zhang G, Terry AV, Bartlett MG. Sensitive liquid chromatography-tandem mass spectrometry method for the determination of the lipophilic antipsychotic drug chlorpromazine in rat plasma and brain tissue. J. Chromatogr. B Analyt. Technol. Biomed. Life Sci. 854, 68-76 (2007).

15 Heller DN. Ruggedness testing of quantitative atmospheric pressure ionization mass spectrometry methods: the effect of co-injected matrix on matrix effects. Rapid Commun. Mass Spectrom. 21(5), 644-652 (2007).

16 Zhang G, Terry AV Jr, Bartlett MG. Determination of the lipophilic antipsychotic drug ziprasidone in rat plasma and brain tissue using liquid chromatography-tandem mass spectrometry. Biomed. Chromatogr. 22(7), 770-778 (2008).

17 Kilcoyne J, Fux E. Strategies for the elimination of matrix effects in the liquid chromatography-tandem mass spectrometry analysis of the lipophilic toxins okadaic acid and azaspiracid-1 in molluscan shellfish. J. Chromatogr. A 1217(45), 7123-7130 (2010).

18 Cartiser N, Bevalot F, Le MC et al. Gas chromatography-tandem mass spectrometry assay for the quantification of four benzodiazepines and citalopram in eleven postmortem rabbit fluids and tissues, with application to animal and human samples. J. Chromatogr. B Analyt. Technol. Biomed. Life Sci. 879(27), 2909-2918 (2011).

19 Dodder NG, Peck AM, Kucklick JR, Sander LC. Analysis of hexabromocyclododecane diastereomers and enantiomers by liquid chromatography-tandem mass spectrometry: chromatographic selectivity and ionization matrix effects. J. Chromatogr. A 1135(1), 36-42 (2006). 